\section{Noisy nociception}

Inner hair cells (IHCs) and outer hair cells (OHCs) in the cochlear organ of Corti (OOC) are destroyed by high-amplitude, persistent sound; however, because the OOC is not innervated by somatosensory nociceptors (which detect damage elsewhere in the body), it is not clear to what extent cochlear damage is detected. Now, Flores et al. show that one set of neurons innervating the OOC responds to noxious sound levels and thus mediates 'auditory nociception'.

Sound-related signals from IHCs are transmitted by type I afferents, whereas OHCs are mostly innervated by type II neurons, the function of which is not known. To determine their role, the authors used mice lacking vesicular glutamate transporter 3 (VGluT3), which is necessary for IHC-type I afferent neurotransmission. Vglut $3^{-/-}$animals exhibited a loss of type I afferent activity (owing to a lack of IHC signalling) and sound-evoked cochlear responses, but their hair cells and type II afferents remained intact.
Next, the authors exposed Vglut $^{-/-}$mice to either loud but harmless sound or sound so intense that it destroyed hair cells. Immunohistochemistry for the neuronal activity marker FOS revealed that, in wild-type mice, exposure to either type of sound induced neuronal activity in the brainstem cochlear nucleus (which receives inputs from the cochlear nerve). By contrast, in Vglut $^{-/-}$mice, cochlear nucleus activity was induced by noxious - but not innocuous - noise, suggesting that there is an alternative cochlea-brain pathway that is distinct from the normal hearing pathway.

The cochlear nucleus response to noxious noise was lost in mice lacking a functional cochlea but with normal vestibular and somatosensory function. This result, together with the finding that noxious noise did not activate vestibular or somatosensory ganglion neurons in either wild-type or Vglut $^{-/-}$mice, indicates that noxious levels of sound are detected by cochlear type II afferents and not by somatosensory or saccular afferents.
Overall, this study shows that type II neurons innervating OHCs in the cochlea mediate auditory nociception. The authors propose that this pathway may be activated not necessarily by sound but by tissue damage caused by noxious sound.

Natasha Bray

ORIGINAL RESEARCH PAPER Flores, E. N et al. A non-canonical pathway from cochlea to brain signals tissue-damaging noise. Curr. Biol. http:// dx.doi.org/10.1016/j.cub.2015.01.009 (2015) in Vglut3 ${ }^{-1-}$

mice, cochlear

nucleus

activity was

induced by

noxious - but

not innocuous

- noise

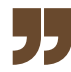

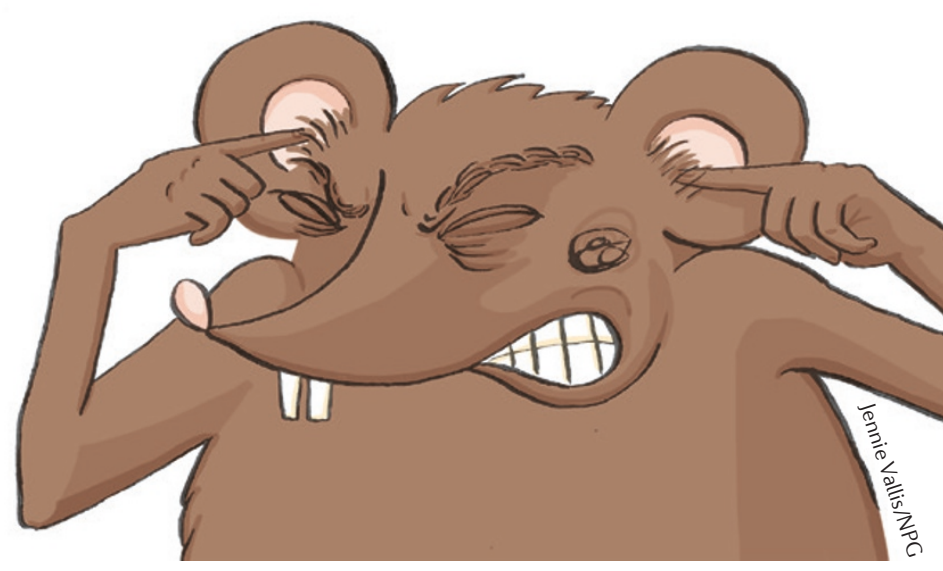

